\title{
Is neuroendocrine differentiation useful to discriminate primary sinonasal intestinal-type adenocarcinomas from metastatic colorectal adenocarcinomas?
}

\author{
Fabrice Projetti, ${ }^{1,2}$ Elie Serrano, ${ }^{2,3}$ Sebastien Vergez, ${ }^{2,4}$ Anne-Charlotte Bissainthe, ${ }^{1,2}$ \\ Marie-Bernadette Delisle, ${ }^{1,5}$ Emmanuelle Uro-Coste ${ }^{1,2}$
}

\begin{abstract}
- Additional material is published online. To view please visit the journal (http:// dx.doi.org/10.1136/jclinpath2014-202463).

For numbered affiliations see end of article.
\end{abstract}

\section{Correspondence to} Fabrice Projetti, Department of Pathology, Universitary Institute of Cancer, 1 Avenue Irène Joliot-Curie, Toulouse 31059, cedex 9, France; projetti.fabrice@gmail.com

Received 3 June 2014 Revised 15 September 2014 Accepted 18 September 2014 Published Online First 7 October 2014

\begin{abstract}
Primary sinonasal intestinal-type adenocarcinomas (ITAC) are defined on the basis of their morphological similarities to colorectal adenocarcinomas (CRA). Thus, differential diagnosis with sinonasal metastasis of CRA could be a real challenge. Neuroendocrine differentiation has been variably described in several types of adenocarcinomas and notably in ITACs and CRAs. In a series of 25 ITACs and 25 lymph node metastasis of CRAs (nmCRA), we analysed neuroendocrine differentiation by immunohistochemistry with antichromogranin A and synaptophysin antibodies. Neuroendocrine differentiation (chromogranin A and/or synaptophysin positivity) was significantly different $(p=0.0002)$ in ITACs $(72 \%)$ and in nmCRAs $(20 \%)$. In conclusion, presence of neuroendocrine cells seems more in favour of a sinonasal intestinal-type adenocarcinoma, than metastatic CRA. This immunohistochemical study could be useful in difficult cases and should be an interesting complement in a clinical discussion.
\end{abstract}

\section{INTRODUCTION}

Primary sinonasal intestinal-type adenocarcinomas (ITAC) are uncommon tumours characterised by morphological and immunohistochemical similarities to colorectal adenocarcinomas (CRA). ${ }^{1}$ Thus, demonstrating differential diagnosis between ITAC and sinonasal metastasis of CRA could be a real challenge, especially in a case with no exposure to wood dust (the main risk factor of ITAC). Sinonasal metastases are rare and occur most frequently in maxillary sinuses. ${ }^{1}$ They correspond to various primary tumours such as renal, lung, breast, thyroid, prostate and CRAs. ${ }^{12}$

Neuroendocrine differentiation, defined by immunohistochemical staining with antichromogranin $\mathrm{A}$ and antisynaptophysin antibodies, has been described in several types of adenocarcinomas. ${ }^{3}$ Some authors suggested that neuroendocrine differentiation was useful for distinguishing ITAC from sinonasal metastatic CRA. ${ }^{4}$ However, neuroendocrine differentiation has been variably reported in ITACs (chromogranin A positivity in $35 \%-75 \%$ of cases and synaptophysin positivity in $0 \%-79 \%)^{4-6}$ and in CRAs (15\%-77\%). ${ }^{7}$ To our knowledge, neuroendocrine markers have been studied in only one series of metastatic CRAs. In this series, ${ }^{3}$ Volante et al showed that metastatic CRAs contained a higher percentage of neuroendocrine cells compared with primary tumours, however, they suggested the possible influence of chemotherapy in agreement with other authors. ${ }^{8}$

We aimed to clarify the usefulness of neuroendocrine differentiation studied by immunohistochemistry with chromogranin $\mathrm{A}$ and synaptophysin antibodies to discriminate primary sinonasal ITAC from metastatic CRAs.

\section{MATERIALS AND METHODS}

We studied 25 ITACs and 25 regional lymph node metastasis of CRAs (nmCRA) from 50 surgical tissue samples corresponding to 50 patients treated between 1999 and 2014 at Toulouse Rangueil University Hospital. This study was performed in accordance with the French bioethics laws having regards to patient information and consent. No patient with ITAC had CRA. No patient had received chemotherapy. Tumours were classified according to WHO classifications. ${ }^{19}$ At the time of resection, all tumour samples were fixed in $4 \%$ formalin, embedded in paraffin and sections stained with H\&E.

Immunohistochemistry analysis was performed with antichromogranin A (clone DAK-A3, dilution 1/400; Dako, Courtaboeuf, France) and antisynaptophysin (clone SP11, dilution 1/200; Thermo Fisher Scientific, Villebon, France) antibodies, on 5 $\mu \mathrm{m}$ thick sections obtained from paraffin-embedded blocks. Slides were processed automatically (Autostainer link 48; Dako) according to the protocols supplied by the antibody manufacturers with the following reagents: FLEX Target Retrieval Solution High, EnVision FLEX Peroxidase-Bloking Reagent, EnVision FLEX Horseradish Peroxidase, EnVision FLEX 3,3'-Diaminobenzidine, EnVision FLEX haematoxylin (Dako).

For each tumour section and the two antibodies, we studied intensity (negative (0), weakly $(1+)$, moderately $(2+)$ and strongly $(3+))$, percentage and pattern (cytoplasmic, nuclear, membrane) of staining. As regards statistical analysis, tumours were classified into two groups: positive (any positive intensity, percentage and pattern of staining) and negative. Neuroendocrine differentiation defined cases with chromogranin A and/or synaptohysin positivity.

We used StatView V.5.0 (SAS Institute, USA software for statistical analyses. Fisher's exact test was employed to assess differences between nominal variables. Results with $\mathrm{p}<0.05$ were considered statistically significant. 
Figure 1 Histological description of a colonic subtype of sinonasal intestinal-type adenocarcinomas (ITAC) with glandular structures and moderate nuclear pleomorphism (A) compared with a moderately differentiated node metastasis of colorectal adenocarcinomas (nmCRA) (D). Scattered positive cells with strong intensity, with antichromogranin $A$ in ITAC (B) and nmCRA (E). Scattered positive cells with weak intensity in ITAC (C) and nmCRA (F). All the scale bars represent $100 \mu$.
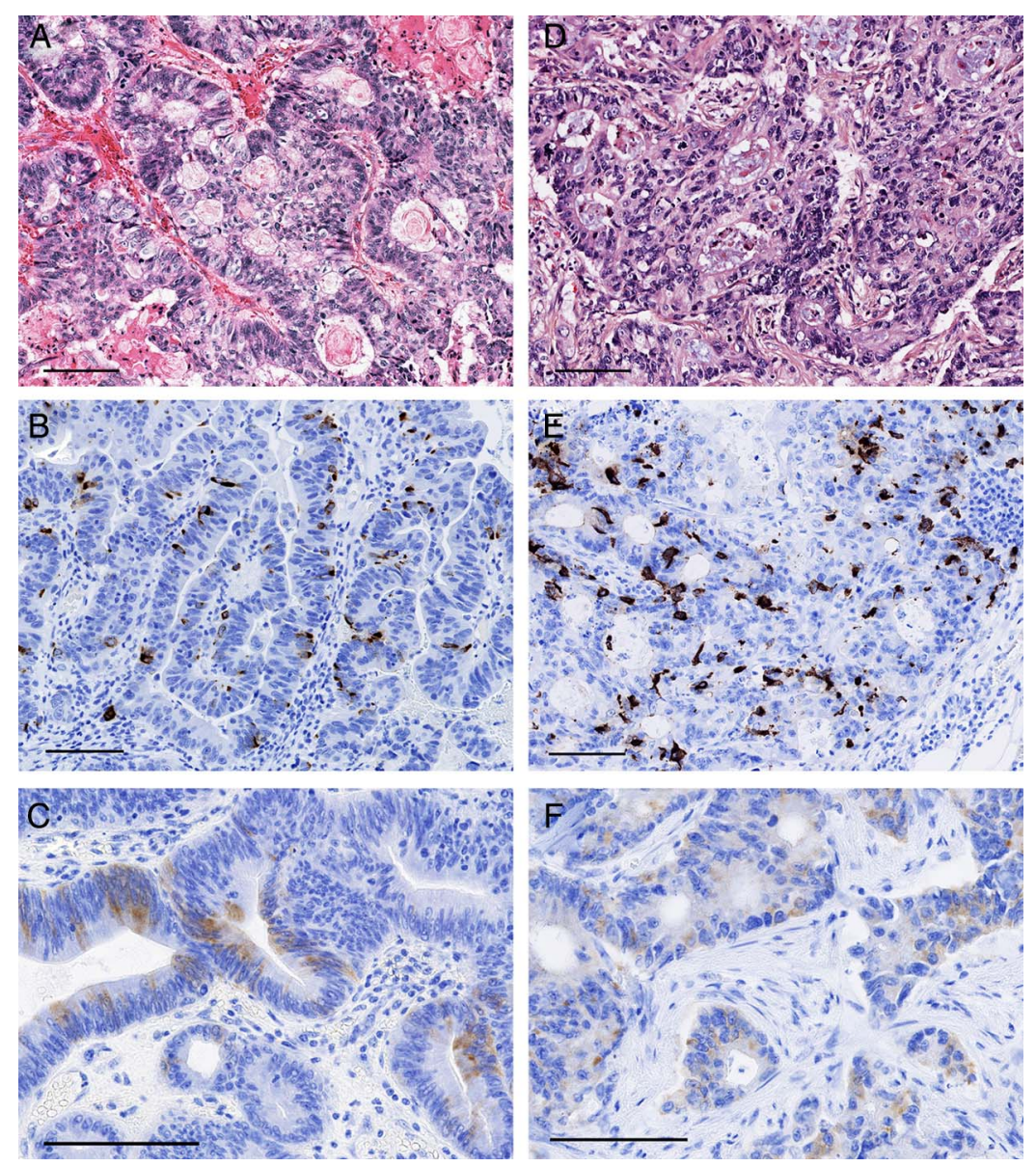

\section{RESULTS}

In the group of ITACs, there were 22 men, three women, and the median age for diagnosis was 67 years (range of 48-91 years). In the group of nmCRAs, there were 13 men, 12 women, and the median age for diagnosis was 66 years (40-86 years). The histological subtypes of ITACs according to WHO classification were papillary (3 cases), colonic (11 cases, figure 1), mucinous ( 7 cases) and mixed (4 cases). The nmCRAs corresponded to 24 conventional adenocarcinomas and one mucinous adenocarcinoma. There were 14 well-differentiated nmCRAs, 8 moderately differentiated (figure 1 ) and 3 poorly differentiated nmCRAs.
Immunohistochemical results according to the tumour type are shown in table 1 . Positivity for chromogranin was shown in $18(72 \%)$ ITACs and $3(12 \%)$ nmCRAs, always with cytoplasmic staining. Positivity for synaptophysin was showed in $14(56 \%)$ ITACs and $3(12 \%)$ nmCRAs, always with cytoplasmic staining. Eighteen (72\%) ITACs and 5 (20\%) nmCRAs showed a neuroendocrine differentiation with chromogranin and/or synaptophysin staining. Immunostaining details of these positive cases are shown in table 2. In most cases, chromogranin A immunostaining was very heterogeneous, scattered, with strong intensity (figure 1 and table 2). In most cases, synaptophysin

Table 1 Immunohistochemical results with antichromogranin A and synaptophysin antibodies according to the tumour type

\begin{tabular}{|c|c|c|c|c|c|c|c|}
\hline \multirow{2}{*}{$\begin{array}{l}\text { Antibodies } \\
\text { Tumour type }\end{array}$} & \multirow[b]{2}{*}{$\mathbf{n}$} & \multicolumn{3}{|c|}{ Chromogranin A } & \multicolumn{3}{|c|}{ Synaptophysin } \\
\hline & & Positive & Negative & p Value* & Positive & Negative & $p$ Value \\
\hline ITACs & 25 & 18 & 7 & $<0.0001$ & 14 & 11 & 0.001 \\
\hline Papillary & 3 & 3 & 0 & & 3 & 0 & \\
\hline Colonic & 11 & 7 & 4 & & 5 & 6 & \\
\hline Mucinous & 7 & 4 & 3 & & 2 & 5 & \\
\hline Mixed & 4 & 4 & 0 & & 4 & 0 & \\
\hline nmCRAs & 25 & 3 & 22 & & 3 & 22 & \\
\hline Well differentiated & $14 \dagger$ & 1 & 13 & & 1 & 13 & \\
\hline Moderately differentiated & 8 & 2 & 6 & & 1 & 7 & \\
\hline Poorly differentiated & 3 & 0 & 3 & & 1 & 2 & \\
\hline
\end{tabular}


Table 2 Tumours showing neuroendocrine differentiation by immunohistochemistry with chromogranin A and/or synaptophysin antibodies according to intensity and percentage of staining

\begin{tabular}{|c|c|c|c|c|c|c|}
\hline \multirow{2}{*}{$\begin{array}{l}\text { Antibodies } \\
\text { Tumour type }\end{array}$} & \multirow[b]{2}{*}{$\mathbf{n}$} & \multicolumn{2}{|c|}{ Chromogranin A } & \multicolumn{2}{|c|}{ Synaptophysine } & \multirow[b]{2}{*}{ p Value* } \\
\hline & & Intensity† & Percentage & Intensity & Percentage & \\
\hline \multirow[t]{2}{*}{ ITACs } & 1 & $3+$ & 1 & $1+$ & $<1$ & \\
\hline & 2 & $3+$ & 15 & $1+$ & 1 & \\
\hline \multirow[t]{17}{*}{$18 / 25(72 \%)$} & 3 & $3+$ & 5 & $1+$ & $<1$ & \\
\hline & 4 & $3+$ & 5 & 0 & 0 & \\
\hline & 5 & $2+$ & 10 & $1+$ & 2 & \\
\hline & 6 & $3+$ & $<1$ & $1+$ & $<1$ & \\
\hline & 7 & $3+$ & 1 & $1+$ & 1 & \\
\hline & 8 & $3+$ & 1 & 0 & 0 & \\
\hline & 9 & $3+$ & $<1$ & 0 & 0 & \\
\hline & 10 & $3+$ & 10 & $2+$ & 2 & \\
\hline & 11 & $3+$ & 1 & $1+$ & $<1$ & \\
\hline & 12 & $3+$ & 1 & 0 & 0 & \\
\hline & 13 & $3+$ & $<1$ & $1+$ & $<1$ & \\
\hline & 14 & $3+$ & 1 & $1+$ & 1 & \\
\hline & 15 & $2+$ & 1 & $1+$ & 1 & \\
\hline & 16 & $3+$ & 1 & $1+$ & $<1$ & \\
\hline & 17 & $1+$ & $<1$ & $1+$ & $<1$ & \\
\hline & 18 & $3+$ & 1 & $1+$ & $<1$ & \\
\hline & & & & & & 0.0002 \\
\hline \multirow[t]{2}{*}{ nmCRAs } & 1 & $2+$ & $<1$ & 0 & 0 & \\
\hline & 2 & 0 & 0 & $1+$ & 90 & \\
\hline \multirow[t]{3}{*}{$5 / 25(20 \%)$} & 3 & $3+$ & 10 & $1+$ & 5 & \\
\hline & 4 & $1+$ & 1 & 0 & 0 & \\
\hline & 5 & 0 & 0 & $2+$ & $<1$ & \\
\hline \multicolumn{7}{|c|}{$\begin{array}{l}{ }^{*} \text { Neuroendocrine differentiation with chromogranin and/or synaptophysin staining was } \\
\text { strongly associated to ITAC in comparison with nmCRAs by Fisher's exact test } \\
(p=0.0002) \text {. } \\
\text { tNegative }(0) \text {, weakly }(1+) \text {, moderately }(2+) \text { and strongly }(3+) \text { staining. } \\
\text { ITACs, intestinal-type adenocarcinomas; nmCRAs, node metastasis of CRA; } n \text {, list of } \\
\text { cases. }\end{array}$} \\
\hline
\end{tabular}

immunostaining was heterogeneous, scattered, with weak intensity (figure 1 and table 2).

Neuroendocrine immunostaining was strongly associated to ITAC in comparison with nmCRAs $(p<0.0001$ for chromogranin; $p=0.001$ for synaptophysin; $p=0.0002$ for chromogranin and/or synaptophysin). There was no link between neuroendocrine immunostaining and histological subtypes and grades.

\section{DISCUSSION}

Differential diagnosis between ITAC and sinonasal metastasis of CRA could be a real challenge, particularly in patients without classical risk factors of ITAC. In these cases, digestive endoscopy and CT scan are usually required to discriminate these two opposite diagnoses. Our study showed a frequent neuroendocrine differentiation (chromogranin $\mathrm{A}$ and/or synaptophysin positivity) in ITACs ( $72 \%$ of cases), compared with only $20 \%$ in nmCRAs. Few authors suggested with scant data, the usefulness of neuroendocrine immunostaining for the differential diagnosis between these two types of tumours. As far as we know, our series is the first comparative study of ITACs and chemonaive nmCRAs. Besides, it strengthens the interest of chromogranin A and synaptopysin immunostaining in the diagnostic discussion.

Chromogranin A and synaptophysin are conventional generic markers of neuroendocrine phenotype, targeting, respectively, neuroendocrine secretory granules and vesicles. Their sensibilities and specificities vary among tumour types. ${ }^{10}$ For example, chromogranin A had a bad sensitivity for paucigranulated neuroendocrine tumours such as rectal carcinoid tumour. ${ }^{10}$ Most of the time, in our series, chromogranin A staining was more intense and more represented in the tumour tissue than synaptophysin staining. Moreover, similar to McKinney et al, ${ }^{5}$ four ITACs had chromogranin A staining without synaptophysin staining. Thus, in accordance with other authors, ${ }^{5}$ chromogranin A appears to be a better marker than synaptophysin to highlight neuroendocrine differentiation in ITACs.

Frequencies of neuroendocrine differentiation in ITACs and nmCRAs observed in our series were consistent with reports of ITACs and primary CRAs. ${ }^{4-7}$ The particular scattered pattern of staining was also similar to others' reports of ITACs, CRAs ${ }^{4} 7$ and other types of adenocarcinomas. ${ }^{10}$ Some authors suggested that this neuroendocrine component was an integral part of the tumour, based on the hypothesis of tumoral pluripotent stem cells. ${ }^{11}$ Our series is in agreement with this concept. Indeed, we showed that some lymph node metastatic CRAs harboured a neuroendocrine component, arguing for their tumoral nature. Prognostic significance of this neuroendocrine differentiation in adenocarcinomas is debated and varies according to the tumour origin. ${ }^{3}{ }^{10}$ Other authors showed a worse clinical outcome in CRA harbouring neuroendocrine cells and suggested the implication of growth factor secretion by these cells, such as vascular endothelial growth factor. ${ }^{11}$ Interestingly, neuroendocrine cells of respiratory tract also secrete growth factors. ${ }^{12}$ Mechanisms, consequences and clinical significance of neuroendocrine differentiation in ITACs remain to be clarified in further studies.

In this comparative study, we have shown that the presence of neuroendocrine differentiation in intestinal-type adenocarcinoma of the sinonasal tract, was better detected by chromogranin A than by synaptophysin and seemed more in favour of a primary tumour than a CRA metastasis. This routinely available immunohistochemical analysis could be part of a beam of arguments including clinical data, abdominal CT scan and, eventually, digestive endoscopy.

\section{Take home message}

- Neuroendocrine differentiation in intestinal-type adenocarcinoma of the sinonasal tract:

- Was better detected by chromogranin A than by synaptophysin by immunohistochemistry.

- And seemed more in favour of a primary sinonasal intestinal-type adenocarcinoma than metastatic colorectal adenocarcinoma.

\section{Author affiliations}

${ }^{1}$ Department of Pathology, Universitary Institute of Cancer, Toulouse, France ${ }^{2}$ Rangueil medical university, Toulouse, France

${ }^{3}$ Department of Otorhinolaryngology-Head and Neck Surgery, Rangueil-Larrey University Hospital, Toulouse, France

${ }^{4}$ Department of Otorhinolaryngology-Head and Neck Surgery, Universitary Institute of Cancer, Toulouse, France

${ }^{5}$ Department of Pathology, Rangueil University Hospital, Toulouse, France

Contributors ES and SV: collected surgical samples and clinical data. FP, EU-C, A-CB: slide interpretation (morphology and immunohistochemistry). FP, EU-C: wrote the manuscript. Solene Evrard: edited the manuscript.

Competing interests None. 
Ethics approval This study was performed in accordance with the French bioethics laws having regards to patient information and consent.

Provenance and peer review Not commissioned; externally peer reviewed.

\section{REFERENCES}

1 Barnes L. Pathology and genetics of head and neck tumours. Lyon: IARC Press, 2005.

2 Donhuijsen $\mathrm{K}$, Hannig $\mathrm{H}$, Schroeder HG, et al. Synchronous adenocarcinomas of intestinal type of the inner nose and the colon. Hum Pathol 2007;38:373-7.

3 Volante M, Marci V, Andrejevic-Blant S, et al. Increased neuroendocrine cells in resected metastases compared to primary colorectal adenocarcinomas. Virchows Arch 2010;457:521-7.

4 Abecasis J, Viana G, Pissarra C, et al. Adenocarcinomas of the nasal cavity and paranasal sinuses: a clinicopathological and immunohistochemical study of 14 cases. Histopathol 2004;45:254-9.

5 McKinney CD, Mills SE, Franquemont DW. Sinonasal intestinal-type adenocarcinoma: immunohistochemical profile and comparison with colonic adenocarcinoma. Mod Pathol 1995;8:421-6.
6 Bashir AA, Robinson RA, Benda JA, et al. Sinonasal adenocarcinoma: immunohistochemical marking and expression of oncoproteins. Head Neck 2003;25:763-71.

7 Pagani A, Papotti M, Abbona GC, et al. Chromogranin gene expressions in colorectal adenocarcinomas. Mod Pathol 1995;8:626-32.

8 Shia J, Tickoo SK, Guillem JG, et al. Increased endocrine cells in treated rectal adenocarcinomas: a possible reflection of endocrine differentiation in tumor cells induced by chemotherapy and radiotherapy. Am J Surg Pathol 2002:26:863-72.

9 Bosman FT. WHO classification of tumours of the digestive system. Lyon: IARC Press, 2010.

10 Lai M, Lu B, Xing X, et al. Secretagogin, a novel neuroendocrine marker, has a distinct expression pattern from chromogranin A. Virchows Arch 2006;449:402-9

11 Gulubova M, Vlaykova T. Chromogranin A-, serotonin-, synaptophysin- and vascular endothelial growth factor-positive endocrine cells and the prognosis of colorectal cancer: an immunohistochemical and ultrastructural study. J Gastroenterol Hepatol 2008:23:1574-85.

12 Jornot L, Lacroix JS, Rochat T. Neuroendocrine cells of nasal mucosa are a cellular source of brain-derived neurotrophic factor. Eur Respir J 2008;32:769-74. 